\title{
Case Report: Persistent Acromegaly - Effective Therapy with Lanreotide and Weekly Pegvisomant Injections
}

\section{(ㄷ) (i) $($ ) $\ominus$}

\author{
Authors \\ Thomas H. Schürmeyer ${ }^{1}$, Britta Galling ${ }^{2,3,4}$
}

Affiliations

1 Klinikum Mutterhaus der Borromäerinnen, Department of Endocrinology, Trier, Germany

2 Charité Universitätsmedizin, Department of Child and Adolescent Psychiatry, Berlin, Germany

3 The Zucker Hillside Hospital, Psychiatry Research, Northwell Health, Glen Oaks, New York, USA

4 Hofstra Northwell School of Medicine at Hofstra University, Hempstead, New York, USA

\section{Key words}

Acromegaly, Growth hormone-secreting pituitary adenoma, Pituitary tumors

received 23.03.2018

revised 30.04.2018

accepted 09.05.2018

\section{Bibliography}

DOI https://doi.org/10.1055/a-0630-0412

Exp Clin Endocrinol Diabetes Rep 2018; 6: e9-e12

(c) J. A. Barth Verlag in Georg Thieme Verlag KG Stuttgart .

New York

ISSN 2196-7407

\section{Correspondence}

Prof. Dr. med. Thomas H. Schürmeyer

Klinikum Mutterhaus der Borromäerinnen

Department of Endocrinology

Feldstraße 16, D-54290 Trier

Germany

Tel.: +49/651/947 2553, Fax: +49/651/9473954

schuermeyer@mutterhaus.de

\begin{abstract}
Objective Therapeutic options for persistent acromegaly after surgery include another surgical approach, radiotherapy, medical therapy or a combination of these procedures. We describe a patient with persistent acromegaly despite heavy pre-treatment who was in need of a simplified mode of effective therapy.

Methods In a 45-year old patient after transsphenoidal pituitary surgery followed by linear accelerator irradiation of the residual tumor two years later acromegaly could not be controlled by a tolerable dosage of intramuscular octreotide LAR. For professional reasons the patient requested a simplified mode of treatment.

Results In order to improve efficacy and treatment convenience, we combined subcutaneous injections of lanreotide up to $120 \mathrm{mg}$ monthly performed by the patient's spouse and of pegvisomant $80 \mathrm{mg}$ weekly conducted by the patient himself. This therapy was well tolerated and satisfactory endocrine control was achieved. Magnetic resonance imaging did not show any remaining tumor within a year. 5 years following surgery and 3 years following irradiation pegvisomant could be stopped and levels of insulin growth factor 1 and growth hormone remained in the normal range. The interval of lanreotide given subcutaneously by the patient's spouse could be extended stepwise and somatostatin analog treatment was terminated after 7.5 years when the patient was cured.

Conclusions Excessive growth hormone secretion persisting after pituitary surgery and irradiation can be controlled in a convenient way for the patient with acromegaly by combining monthly lanreotide and weekly pegvisomant injections, if monotherapy with a somatostatin analog fails.
\end{abstract}

\section{Introduction}

Patients with acromegaly due to a growth hormone $(\mathrm{GH})$ secreting pituitary adenoma suffer from physical changes and a lower life expectancy [1]. The primary goal of treatment is a reduction of $\mathrm{GH}$ production and normalization of insulin-like growth factor 1 (IGF-1) levels. Elevated IGF-1 level elevations coincide with a reduced quality of life due to persistent acromegaly and a considerably increased mortality [1]. There is evidence, that long-term results of the neurosurgery can be positively influenced by pretreatment with somatostatin analogs (SSA) [2]. In about $60 \%$ of the patients neurosurgical resection of the tumor normalizes excessive $\mathrm{GH}$ secretion and the serum level of insulin-like growth factor-1 (IGF-1) $[1,3]$. Routine use of preoperative medical treatment is not recommended, but therapy with SSA can reduce surgical risks from comorbidities [4]. 
Therapeutic options for persistent acromegaly after the surgery include another surgical approach, radiotherapy, medical therapy (SSA, PEGV, dopamine agonists) or a combination of these procedures. However, in 65-83\% of patients persistent IGF-1 normalization cannot be achieved by SSA monotherapy [4]. In these cases, combination therapy with daily PEGV injections can be applied to improve treatment outcome $[1,3,5,6]$ resulting in a normalization of the IGF-1 level, decreased symptoms of acromegaly, as well as an improved quality of life [7-9]. However, combination therapy with PEGV is not only expensive, but moreover requires the patients' compliance and ability to perform daily subcutaneous injections according to the approved dosing schedule.

This case report presents a patient with persistent acromegaly after transsphenoidal surgery and linear accelerator irradiation (LINAC) of a residual tumor who was in need of a simplified mode of combination therapy. Since for professional reasons the patient could not see a physician every month, his spouse was trained to give subcutaneous injections of the SSA lanreotide.

\section{Case Report}

\section{Description of the patient}

In 2009, the 45-year-old, caucasian male presented with a continuous growth of his hands and feet starting about 20 years ago. In addition, extreme snoring, an increase of weight from 106 to $120 \mathrm{~kg}$ in about 5 years, a carpal tunnel syndrome of the left hand, diabetes mellitus type 2 (HbA1c $6.1 \%$ [43 mmol/mol]), arterial hypertension, colon polyps and a hypertrophic pharynx uvula causing a reinforced gagging reflex were observed. Visual fields were normal.

\section{Initial diagnostics}

In May 2009 cerebral magnetic resonance imaging (MRI) revealed a $22 \mathrm{~mm}$ pituitary tumor invading the right cavernous sinus. Laboratory results showed elevated serum levels of IGF-1 up to $1,085 \mathrm{ng} /$ $\mathrm{mL}$ (upper normal value $246 \mathrm{ng} / \mathrm{mL}$, CLIA Immulite $2000 \circledR$ Sie-

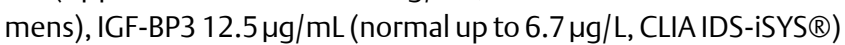
and $\mathrm{GH}\left(20.5 \mu \mathrm{g} / \mathrm{L}, \mathrm{CLIA}\right.$ Liason ${ }^{\circledR}$ DiaSorin). Total and SHBG corrected plasma and SHBG-corrected testosterone was slightly decreased but thyreotropic and adrenocorticotropic pituitary functions were found to be normal.

\section{Therapeutic interventions and results}

The results of various therapeutic approaches from 2009 to 2017 on IGF-1 levels are shown in > Fig. 1. Therapy with octreotide LAR $20 \mathrm{mg} / 4$ weeks i.m. was started in advance of the pituitary surgery that was performed in February 2010. Following transsphenoidal endoscopic resection of the tumor, acromegaly persisted so that treatment with octreotide LAR was continued, but without normalizing the IGF-1 values. Residues of the tumor located in the syphon of the right carotid artery could be demonstrated by MRI in June 2010. A trial of increasing the dosage of octreotide LAR was not tolerated by the patient due to persistent diarrhea. $0,5 \mathrm{mg}$ of cabergoline caused nausea, dizziness and hallucinations so that the patient refused to continue this kind of therapy. Finally, a monthly injection of octreotide LAR $20 \mathrm{mg}$ was combined with daily application of PEGV (Somavert ${ }^{\circledR}$ ) $10 \mathrm{mg}$ starting in September 2010 , resulting in a normalization of IGF- 1 levels. Since PEGV therapy interferes with the measurement of STH, the required dose of SSA and PEGV was adjusted on the basis of IGF1-levels exclusively.

However, for professional reasons the patient requested a simplification of the mode of therapy. Therefore, in November 2010, octreotide was replaced by lanreotide (Somatuline Autoge ${ }^{\circledR}$ ) $90 \mathrm{mg} / 4$ weeks and the patient's spouse was trained to give subcutaneous injections. 3 months later, in January 2011, the daily s.c. injection of $10 \mathrm{mg}$ PEGV administration was replaced by a modified dosing schedule of $80 \mathrm{mg}$ PEGV once weekly to further simplify the therapy. Under these treatment modifications IGF-1 levels were within or slightly above the upper limit of the normal range. To treat the residual adenoma the patient underwent LINAC radiosurgery in December 2011 (linear accelerator, focal dosage of $176 \mathrm{~Gy}$ ). As IGF-1 levels value remained at or above the upper limit of normal, the lanreotide dose had to be increased to $120 \mathrm{mg} / 4$ weeks in November 2011 while weekly PEGV administration was maintained ( $\triangleright$ Fig. 1).

By this mode of combination therapy biochemical control of acromegaly was achieved as well as an improvement of many of the patient's symptoms. MRI of the pituitary region 1,2 and $31 / 2$ years following the irradiation therapy did not show further residual pituitary tumor growth ( $\triangleright$ Fig. 2 ).

The patient was very satisfied with the simplified therapeutic regime performed by himself with the aid of his spouse as for professional reasons he could not see a physician at frequent intervals and was not willing to receive daily injections. No adverse or unexpected events occurred under this mode of treatment. In 2014 the interval between lanreotide injections could be extended to 6 weeks and in March 2015, 3 years and 3 months following irradiation therapy, PEGV treatment could be discontinued. 6 months later, in October 2015, IGF-1 $(214 \mathrm{ng} / \mathrm{mL})$ and $\mathrm{GH}(0.48 \mu \mathrm{g} / \mathrm{L}) \mathrm{lev}$ els continue to be normal. In June 2016 the interval of lanreotide injections was extended to 8 weeks and in December 2016 lanreo-

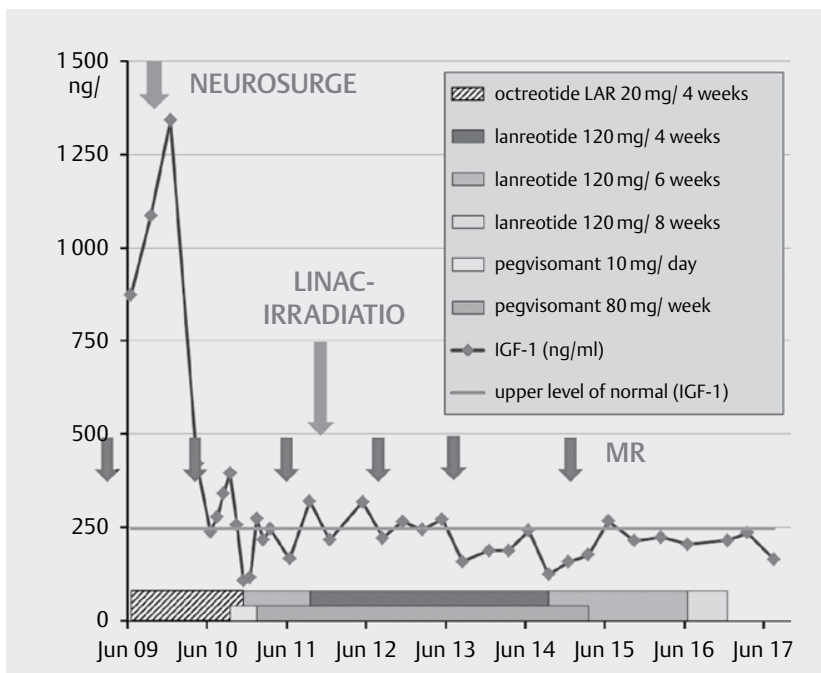

- Fig. 1 IGF-1 levels in a patient with acromegaly under various therapeutic approaches. IGF-1: insulin-like growth factor 1, LINAC: linear accelerator. 
tide treatment was discontinued. The patient's follow-up showed normal levels of IGF- 1 (215 and $235 \mathrm{ng} / \mathrm{mL})$ and $\mathrm{GH}(0.27 \mu \mathrm{g} / \mathrm{L}$ and $0.37 \mu \mathrm{g} / \mathrm{L}) 3$ and 6 months later. During the treatment period thyreotropic, adrenocorticotropic and gonadotropic pituitary function was controlled every six months. Initially testosterone was replaced by injections of testosterone undecanoate ( $1000 \mathrm{mg} q 3$ months), which in 2015 was replace by testogel $₫ 50$ mg daily. As gonadotropic function normalized testosterone replacement could be discontinued in December 2016. All other pituitary functions stayed at normal throughout the observation period.

\section{Discussion}

In contrast to octreotide LAR which has to be injected intramuscularly lanreotide can be injected subcutaneously, so that injections can be performed by trained laymen without professional help [1012]. The efficacy of long therapeutic intervals was shown in trials with lanreotide autogel $[13,14]$ and it is well known that a shift from octreotide to lanreotide has a similar effectiveness and tolerability [13]. The combination of SSA and PEGV is a useful and safe option when monotherapy is insufficient. It is effective in $97 \%$ of the patients $[15,16]$. A 28-week trial combining lanreotide and PEGV therapy in acromegalic patients resulted in the normalization of the IGF-1 value and in clinical improvements in $57.9 \%$ of the patients that had been insufficiently controlled by SSA or PEGV monotherapy $[7,17]$. Weekly administration of PEGV is effective in controlling serum IGF- 1 levels in acromegaly and the regimen of weekly dosing is preferred by many patients $[18,19]$.

In patients with diabetes mellitus efficacy of combining SSA and PEGV therapy seems to be slightly decreased [7]. Hepatic enzymes should be monitored regularly in all patients treated with PEGV [9]. In our patient the HbA1c level normalized and transaminases were within normal ranges irrespective of the therapeutic regimen used.

\section{Conclusion}

This case shows that in patients with acromegaly excessive $\mathrm{GH}$ secretion persisting following pituitary surgery and irradiation can be controlled by a combination therapy of monthly lanreotide and weekly PEGV injections, if monotherapy with SSA fails. This mode
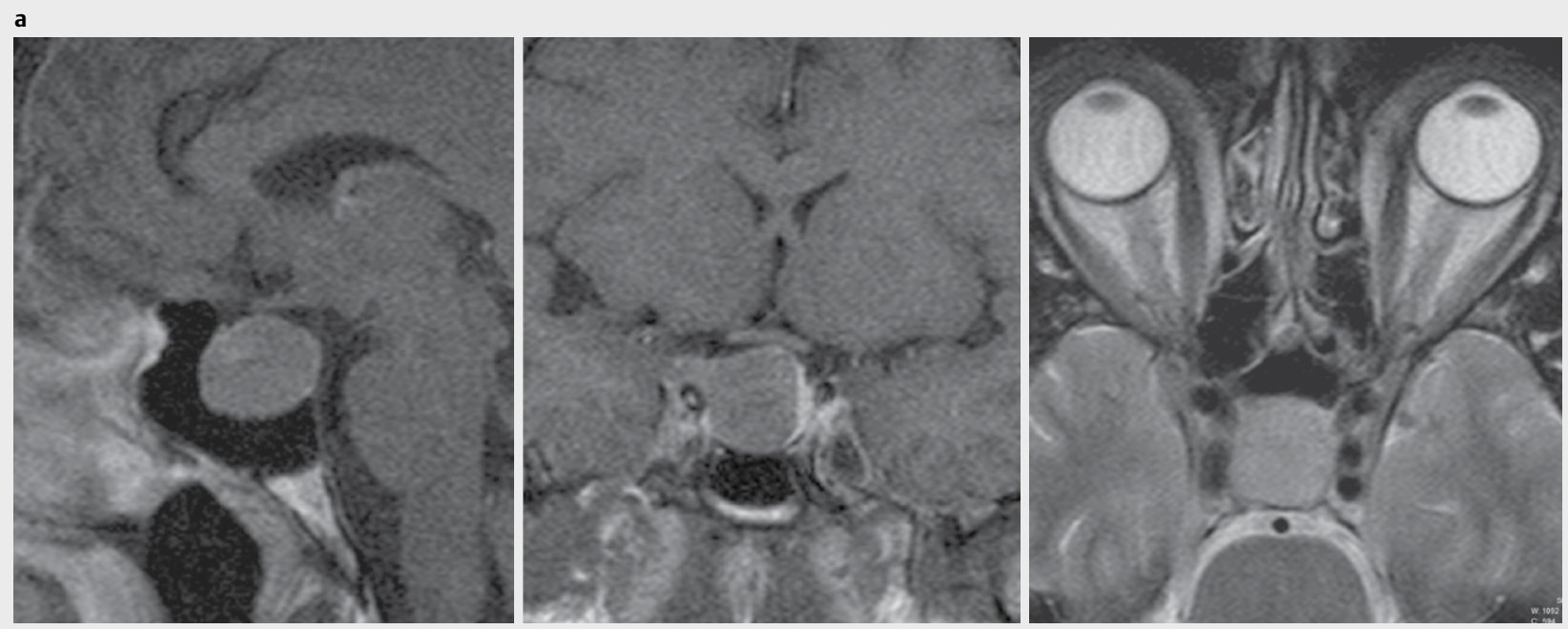

b
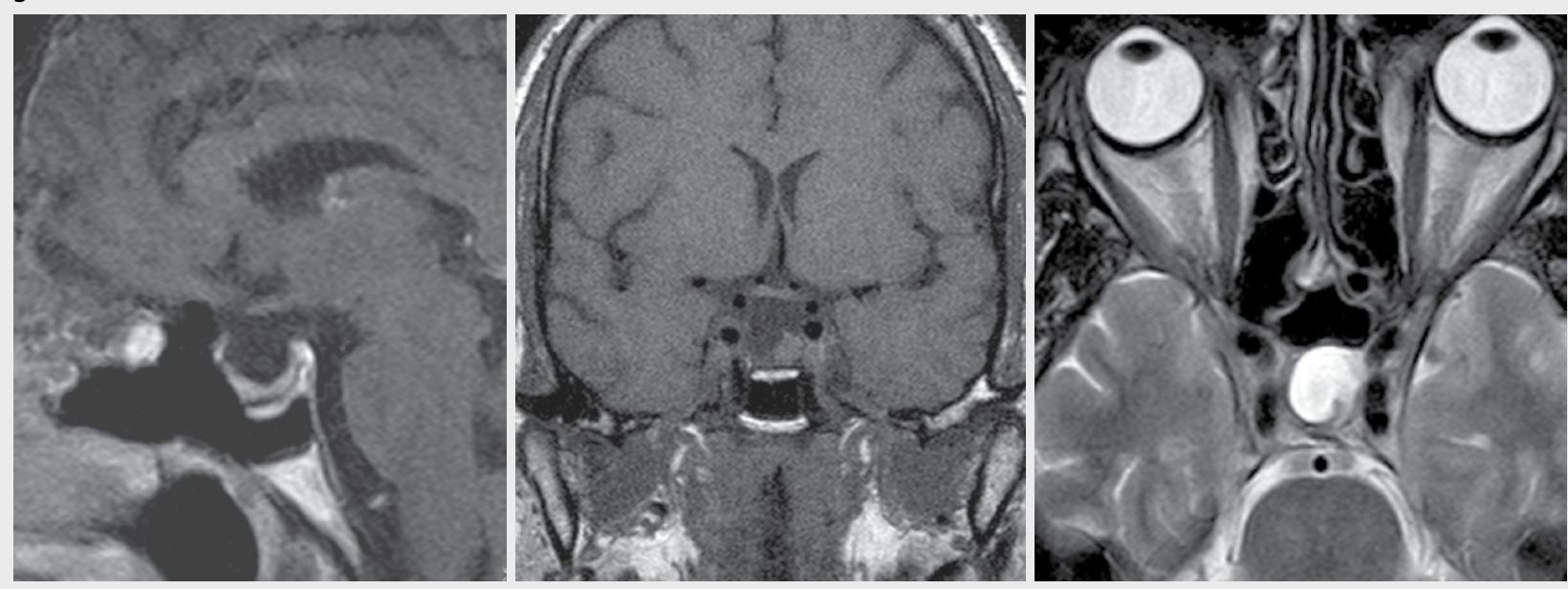

Fig. 2 MR imaging of the pituitary region a at diagnosis and $\mathbf{b} 3$ years following transsphenoidal surgery, 1 year following LINAC irradiation MR: magnetic resonance, LINAC: linear accelerator. 
of treatment can be performed by lay people or the patient himself without professional help. A period of several years can be bridged satisfactorily until radiation therapy has the desired effect. After 1 year of radiation therapy no residual tumor growth was detectable by MRI. However, a biochemical cure was only achieved after 5 years. During this treatment period, PEGV was discontinued after 3 years while lanreotide was phased out and finally discontinued 5 years after LINAC therapy. No side effects of radiation therapy were observed at the last visit, although hypopituitarism may still occur after longer follow-up. Currently the patient is in complete remission.

\section{Conflict of Interest}

Professor Dr. Schürmeyer and Dr. Galling received funding for editorial support, provided by Dr. Joachim Sauer, from Ipsen Pharma GmbH.

\section{References}

[1] Feelders RA, Hofland L], van Aken MO et al. Medical therapy of acromegaly: Efficacy and safety of somatostatin analogues. Drugs 2009; 69: 2207-2226

[2] Pita-Gutierrez F, Pertega-Diaz S, Pita-Fernandez $S$ et al. Place of preoperative treatment of acromegaly with somatostatin analog on surgical outcome: A systematic review and meta-analysis. PLoS One 2013; 8: e61523

[3] Andersen M. Management of endocrine disease: $\mathrm{GH}$ excess: Diagnosis and medical therapy. Eur J Endocrinol 2014; 170: R31-R41

[4] Katznelson L, Laws ER Jr., Melmed S et al. Acromegaly: An endocrine society clinical practice guideline. J Clin Endocrinol Metab 2014; 99: 3933-3951

[5] Fleseriu M. The role of combination medical therapy in acromegaly: Hope for the nonresponsive patient. Curr Opin Endocrinol Diabetes Obes 2013; 20: 321-329

[6] Wang JW, Li Y, Mao ZG et al. Clinical applications of somatostatin analogs for growth hormone-secreting pituitary adenomas. Patient Prefer Adherence 2014; 8: 43-51

[7] van der Lely AJ, Bernabeu I, Cap J et al. Coadministration of lanreotide Autogel and pegvisomant normalizes IGF1 levels and is well tolerated in patients with acromegaly partially controlled by somatostatin analogs alone. Eur J Endocrinol 2011; 164: 325-333
[8] Neggers S], van Aken MO, de Herder WW et al. Quality of life in acromegalic patients during long-term somatostatin analog treatment with and without pegvisomant. J Clin Endocrinol Metab 2008; 93: 3853-3859

[9] Neggers S], de Herder WW, Janssen JA et al. Combined treatment for acromegaly with long-acting somatostatin analogs and pegvisomant: Long-term safety for up to 4.5 years (median 2.2 years) of follow-up in 86 patients. Eur J Endocrinol 2009; 160: 529-533

[10] Bevan JS, Newell-Price J, Wass JA et al. Home administration of lanreotide Autogel by patients with acromegaly, or their partners, is safe and effective. Clin Endocrinol (Oxf) 2008; 68: 343-349

[11] Salvatori R, Nachtigall LB, Cook DM et al. Effectiveness of self- or partner-administration of an extended-release aqueous-gel formulation of lanreotide in lanreotide-naive patients with acromegaly. Pituitary 2010; 13: 115-122

[12] Salvatori R, Woodmansee WW, Molitch M et al. Lanreotide extendedrelease aqueous-gel formulation, injected by patient, partner or healthcare provider in patients with acromegaly in the United States: 1-year data from the SODA registry. Pituitary 2014; 17: 13-21

[13] Schopohl J, Strasburger C], Caird D et al. Efficacy and acceptability of lanreotide Autogel(R) $120 \mathrm{mg}$ at different dose intervals in patients with acromegaly previously treated with octreotide LAR. Exp Clin Endocrinol Diabetes 2011; 119: 156-162

[14] Adelman DT, Liebert KJ, Nachtigall LB et al. Acromegaly: The disease, its impact on patients, and managing the burden of long-term treatment. Int J Gen Med 2013; 6: 31-38

[15] Neggers SJ, van Aken MO, Janssen JA et al. Long-term efficacy and safety of combined treatment of somatostatin analogs and pegvisomant in acromegaly. J Clin Endocrinol Metab 2007

[16] Puig-Domingo M, Soto A, Venegas E et al. Use of lanreotide in combination with cabergoline or pegvisomant in patients with acromegaly in the clinical practice: The ACROCOMB study. Endocrinol Nutr 2016; 63: 397-408

[17] van der Lely AJ, Muller A, Janssen JA et al. Control of tumor size and disease activity during cotreatment with octreotide and the growth hormone receptor antagonist pegvisomant in an acromegalic patient. J Clin Endocrinol Metab 2001; 86: 478-481

[18] Higham CE, Thomas JD, Bidlingmaier M et al. Successful use of weekly pegvisomant administration in patients with acromegaly. Eur J Endocrinol 2009; 161: 21-25

[19] Neggers S], de Herder WW, Feelders RA et al. Conversion of daily pegvisomant to weekly pegvisomant combined with long-acting somatostatin analogs, in controlled acromegaly patients. Pituitary 2011; 14: 253-258 